\title{
Microscopic Description of Rotating Magnetocaloric Effect in Frustrated Antiferromagnetic System $\mathrm{TmB}_{4}$
}

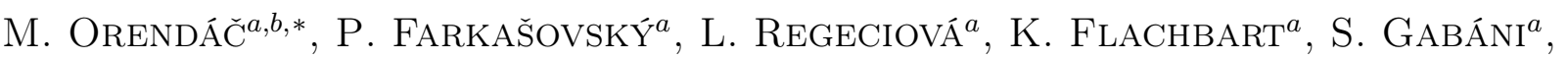 \\ E. GaŽO ${ }^{a}$, G. Pristáš ${ }^{a}$, A. Dukhnenko ${ }^{c}$, N. Shitsevalova ${ }^{c}$ And K. Siemensmeyer ${ }^{d}$ \\ ${ }^{a}$ Institute of Experimental Physics, Slovak Academy of Sciences, Watsonova str. 47, 04001 Košice, Slovakia \\ ${ }^{b}$ Institute of Physics, Faculty of Science, P.J. Safarik University, Park Angelinum str. 9, 04154 Košice, Slovakia \\ ${ }^{c}$ Institute for Problems of Materials Science, National Academy of Sciences of Ukraine, \\ Krzhyzhanovsky str. 3, $03680 \mathrm{Kiev}$, Ukraine \\ ${ }^{d}$ Helmholtz-Zentrum Berlin, Glienicker str. 100, 14109 Berlin, Germany
}

\begin{abstract}
$\mathrm{TmB}_{4}$ is an anisotropic magnetic system with geometrical frustration of Shastry-Sutherland type. Experimentally, it was the rotating magnetocaloric effect (R-MCE) of $\mathrm{TmB}_{4}$ evaluated from measurements of temperature dependences of heat capacity in various magnetic fields and crystallographic orientations. R-MCE in this system shows a complex behavior of the adiabatic temperature change (AdT) - cooling above $T_{\mathrm{N}}$ and two heating regions in ordered phase [1]. In this contribution, a theoretical model with spin-electron Hamiltonian was suggested to explain the complex AdT behavior. The model is based on the idea of two interacting subsystems: the localized spins of rare earth ions, and the itinerant electrons in conduction band. The received results from Monte Carlo approach successfully reproduce the observed heating and cooling regions. Thus, our study shows that measurements of R-MCE can be an effective tool for investigation of the microscopic properties of magnetization processes.
\end{abstract}

DOI: 10.12693/APhysPolA.137.764

PACS/topics: tetraborides, Rotating-Magneto-Caloric effect, Monte Carlo

\section{Introduction}

The magnetocaloric effect (MCE) is a magnetothermodynamic phenomenon in which temperature variation in magnetic material is caused by the change of external magnetic field [1-3]. It was also shown that geometrical spin frustration significantly enhances the change of magnetic entropy in applied magnetic field, and thus intensify the MCE $[1,4]$. In the case of strongly anisotropic systems the rotating magnetocaloric effect (R-MCE) can be used, as proposed and investigated in $[1,2,5,6]$. However, the MCE can be also obtained in anisotropic systems by simply rotating the magnetic refrigerant in constant field instead of moving it in and out of the magnet [1]. Thulium tetraboride $\left(\mathrm{TmB}_{4}\right)$ is an anisotropic geometrically frustrated magnetic system. It belongs to the group of rare earth tetraborides $\left(\mathrm{REB}_{4}\right)$ that crystallize in a tetragonal lattice. As one of the three valence electrons of rare earth $\mathrm{RE}^{3}+$ ions goes to the conduction band, these tetraborides are good metals, and the interaction between the localized magnetic ions and the itinerant conduction electrons is playing an important role [1]. In case of $\mathrm{TmB}_{4}$ the magnetic $\mathrm{Tm}^{3}+$ ions have a $4 f^{12}$ configuration with an angular momentum $J=6$. In the mentioned tetragonal lattice the Tm ions lie in sheets perpendicular to the $c$-axis, and can be within the $a-b$ plane mapped onto the frustrated Shastry-Sutherland lattice, which can be viewed in terms

\footnotetext{
* corresponding author
}

of squares and equilateral triangles $[1,7]$. Between these $\mathrm{Tm}$ sheets there are planes of boron atoms grouped into $\mathrm{B}_{6}$ octahedra and dimer pairs. Crystal field effects at $\mathrm{Tm}^{3+}$ sites lift the degeneracy of $J=6$ multiplet. Consequently, the ground state is a doublet $M_{J}= \pm 6$, which induces a strong Ising-like magnetic anisotropy with magnetic moments of $\mathrm{Tm}$ ions oriented along the $c$-axis below its Néel temperature $T_{\mathrm{N}}=11.7 \mathrm{~K}$. In the ordered antiferromagnetic state the magnetization $M$ for magnetic fields $\boldsymbol{B} \| c$ reaches saturation $M_{S}$ at about $4 \mathrm{~T}$ accompanied by magnetization plateaus at $\frac{1}{2} M_{S}$ and $\frac{1}{8} M_{S}[1,7]$. On the other hand, for fields $\boldsymbol{B} \perp c$ the saturation of $M$ is reached only at fields above $30 \mathrm{~T}[1,8]$. Thus, it follows that in magnetic fields up to about $4 \mathrm{~T}$ the magnetization along the $c$-axis is considerably higher than this in the perpendicular direction which is advantageous for the emergence of the R-MCE [1]. Recently, the R-MCE in $\mathrm{TmB}_{4}$ was experimentally evaluated [1]. According to reported procedure, at first temperature dependencies of heat capacity $C(T, B)$ at various fields were measured in orientations $\boldsymbol{B} \| c$ and $\boldsymbol{B} \perp c$ up to $4.8 \mathrm{~T}$. It was shown that due to the high anisotropy of $\mathrm{TmB}_{4}$ the heat capacity in $\boldsymbol{B} \perp c$ was field independent (up to $4.8 \mathrm{~T}$ ). Then, the entropy distributions $S(T, B)$ were calculated in both field orientations using the well known formula [1]:

$$
S(T)_{H}=\int_{0}^{T} \frac{C(T)_{H}}{T} \mathrm{~d} T+S_{0, H} .
$$

The entropy distribution in the field direction $\boldsymbol{B} \perp c$ are straight lines along $\boldsymbol{B}$ axis $\left(C_{\boldsymbol{B} \perp c}(T)\right.$ does not depend on $\boldsymbol{B})$, see [1]. On the other hand, the entropy map in 


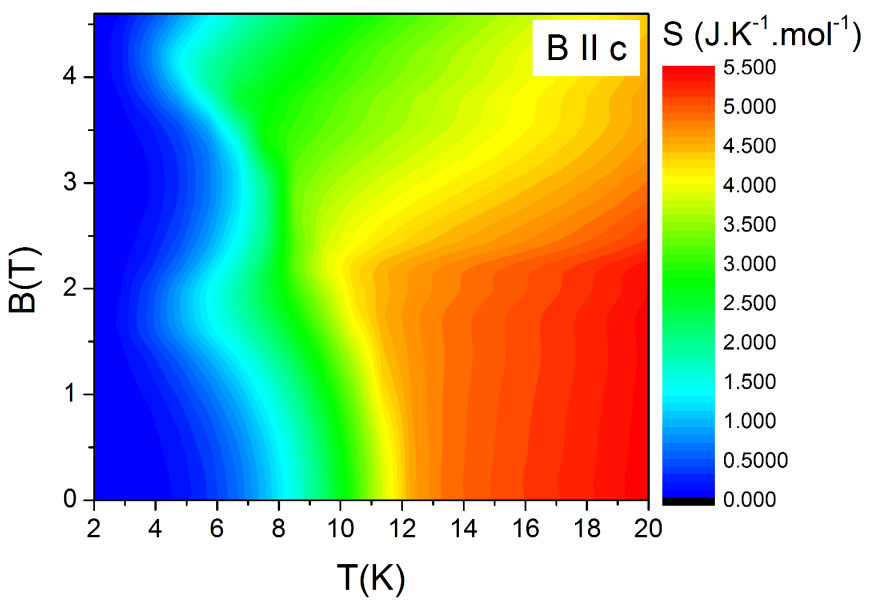

Fig. 1. Entropy distribution for $\boldsymbol{B} \| c$ calculated from temperature dependencies of heat capacity $C(T, B)[1]$.

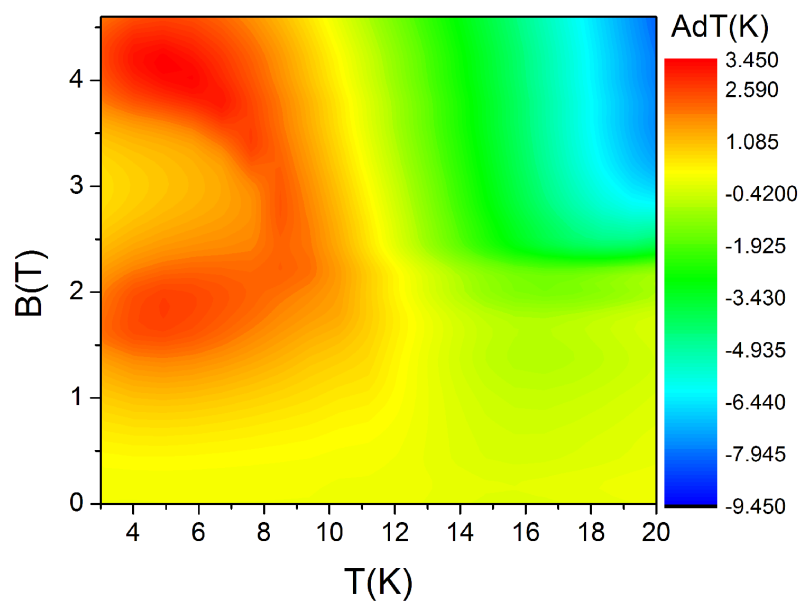

Fig. 2. Layout of the adiabatic temperature change $\operatorname{AdT}(T, B)$ determined from $S(T, B)$ distributions using (2) [1].

the field direction $\boldsymbol{B} \| c$ shows much more complexity, see Fig. 1. Note that the contours of the entropy distribution for $\boldsymbol{B} \| c$ display rather well the $B-T$ phase diagram of $\mathrm{TmB}_{4}$. The $B-T$ phase diagram, as well as details of measurement and entropy calculations can be found in [1].

Next, the adiabatic temperature change AdT can be calculated using the formula [1]:

$$
\operatorname{AdT}(T, B)=T(S)_{B \perp c}-T(S)_{B \| c} .
$$

Distribution of AdT given by (2) is shown in Fig. 2 (for full range plot up to $60 \mathrm{~K}$, see [1]). It exhibits a large cooling region above $T_{\mathrm{N}}$ (around $20 \mathrm{~K}$ and in fields above $2 \mathrm{~T}$ ) in which the temperature of $\mathrm{TmB}_{4}$ during the rotation decreases by more than $9 \mathrm{~K}$ (this cooling procedure is analogous to the conventional demagnetization process in the paramagnetic region). But, there is also a positive (warming up) area below $T_{\mathrm{N}}$ (around $5 \mathrm{~K}$ at $1.8 \mathrm{~T}$ and $4.2 \mathrm{~T}$ ), where the temperature increases by more than $2.5 \mathrm{~K}$ when the sample is rotated from $\boldsymbol{B} \| c$ to $\boldsymbol{B} \perp c$, and which is related with heating at magnetic reversal in the ordered state [1]. In our contribution, we present the microscopic description of observed heating and cooling phenomenon.

\section{Model}

For data interpretation the used model was proposed for explanation of magnetization processes in rare-earth tetraborides [9]. It is based on the idea of two interacting subsystems: the localized spins of rare earth ions, and the itinerant electrons in the conduction band. The localized spins are described by following Hamiltonian [9]:

$$
H_{J_{1} J_{2}}=J_{1} \sum_{i j} S_{i}^{z} S_{j}^{z}+J_{2} \sum_{i j} S_{i}^{z} S_{j}^{z}-h \sum_{i} S_{i}^{z},
$$

which describes interactions of the localized spins $S_{i}^{z}$ with the first and second nearest neighbors on the SSL with interaction strengths $J_{1}, J_{2}$, and external magnetic field $h$. Taking into account the conduction electrons this "starting" Hamiltonian was expanded with additional terms [9]:

$$
\begin{aligned}
H & =\sum_{i j \sigma} t_{i j} d_{i \sigma}^{\dagger} d_{j \sigma}+J_{z} \sum_{i}\left(n_{i \uparrow}-n_{i \downarrow}\right) S_{i}^{z} \\
& -h \sum_{i}\left(n_{i \uparrow}-n_{i \downarrow}\right)+H_{J_{1} J_{2}},
\end{aligned}
$$

where $d_{i \sigma}^{\dagger}, d_{i \sigma}$ are the creation and annihilation operators of the itinerant electrons in the $d$-band Wannier state at site $i$, and $n_{i \sigma}=d_{i \sigma}^{\dagger} d_{i \sigma}$. The first term of (4) describes the kinetic energy of the itinerant $d$ electrons, that can move between sites via the hopping mechanism. The probability of the intersite transition is described by matrix elements $t_{i j}$, which are $-t$ if $i$ and $j$ are the nearest neighbors, $-t^{\prime}$ if $i$ and $j$ are the next-nearest neighbors from the SSL, and zero otherwise. The electron and spin subsystems interact only via the spin-dependent Ising interaction $J_{z}$, which is represented by second term. The third term is an interaction between the external magnetic field and the itinerant electrons [9]. With the use of Monte Carlo (MC) approach, we were able to solve (4) numerically. From the MC simulation temperature dependencies of the heat capacity at various fields (in $\boldsymbol{B} \| c$ direction) were obtained. It should be noted that the lattice contribution to the heat capacity was neglected, because the results of the model and the experiment will be compared only at low temperatures, where magnetic and electron contributions are dominant. The heat capacity in the $\boldsymbol{B} \perp c$ direction was obtained with the following approach. Supposing that the system is infinitely anisotropic (saturation field in $\boldsymbol{B} \| c$ is $4 \mathrm{~T}$, while in $\boldsymbol{B} \perp c$ it is more than $30 \mathrm{~T}$ [8]), the field effectively acting on the system will be a projection of $\boldsymbol{B}$ into $c$ axis. In the case of $\boldsymbol{B} \perp c$ for any value of field projection in $c$ axis is zero. This approach yields the result that the heat capacity for any non-zero field will be same as the heat capacity in zero field. In the zero field 


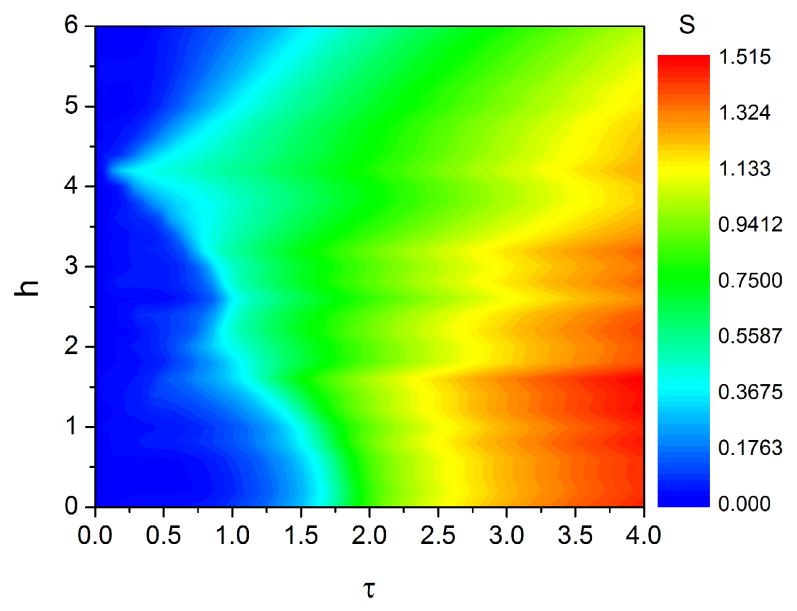

Fig. 3. Entropy distribution for $\boldsymbol{B} \| c$ calculated from temperature dependencies of heat capacity obtained from model (4).

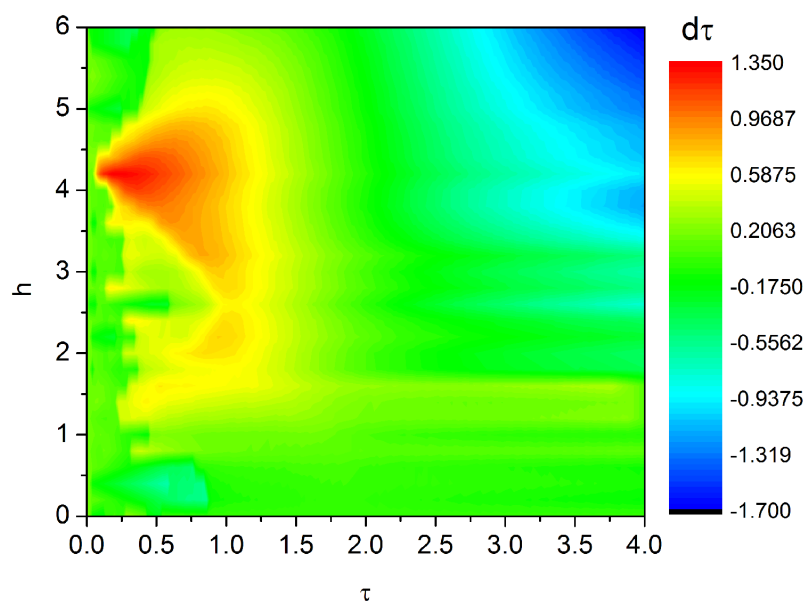

Fig. 4. Layout of the adiabatic temperature change $\operatorname{AdT}(T, B)$ determined from theoretical $S(T, B)$ (Fig. 3) distributions using (2).

the heat capacity is independent of field orientation so $C_{\boldsymbol{B} \perp c}(T, B)=C_{\boldsymbol{B} \perp c}(T, B=0)$. The R-MCE evaluation procedure was same as in experimental case. The entropy distribution in Fig. 3 was calculated using (1).

The entropy distribution (Fig. 3) behaves qualitatively the same as one obtained from the experiment (see Fig. 1). Also, the contour of the experimental $B-T$ phase diagram is visible. The noise that is present here, is probably caused by the finite lattice size effects and the numerical errors. The distribution of AdT was calculated using (2), and it is shown on Fig. 4.

Comparing the theoretical (Fig. 4) and experimental distribution (Fig. 2) of AdT, one can observed a qualitative match: one cooling region at higher temperatures and two heating regions at lower temperatures. Again the finite lattice size effects, and numerical errors manifest as noise especially at low temperatures.

\section{Conclusions}

In summary, we have applied the electron-spin model, used previously for explanation of magnetization processes in rare earth tetraborides, to qualitatively describe the R-MCE in $\mathrm{TmB}_{4}$. From the model simulation the heat capacity data were obtained. Using the same procedure of evaluation of R-MCE as in the experimental case, the distributions of entropy and AdT were calculated. Comparing theoretical results with experimental ones, the qualitative match was achieved, which justifies used model and methods.

\section{Acknowledgments}

This work was supported by the project VEGA 2-0032-16, by the Slovak Research and Development Agency under the contract No. APVV 17-0020, project DAAD-SAS, project ITMS 26230120002 and ITMS project ITMS 26210120002. Liquid nitrogen for experiments was sponsored by U.S. Steel Kosice.

\section{References}

[1] Mat. Orendáč, S. Gabáni, E. Gažo, G. Pristáš, N. Shitsevalova, K. Siemensmeyer, K. Flachbart, Sci. Rep. 8, 10933 (2018).

[2] M.D. Kuzmin, A.M. Tishin, J. Phys. D: Appl. Phys. 24, 2039 (1991).

[3] K.A. Gschneidner Jr., V.K. Pecharsky, A.O. Tsokol, Rep. Prog. Phys. 68, 1479 (2005).

[4] M.E. Zhitomirsky, Phys. Rev. B 67, 104421 (2003).

[5] H. Zhang, Y.W. Li, E. Liu, Y.J. Ke, J.L. Jin, Y. Long, B.G. Shen, Sci. Rep. 5, 11929 (2015).

[6] V. Tkáč, A. Orendáčová, E. Čižmár, M. Orendáč, A. Feher, A.G. Anders, Phys. Rev. B 92, 024406 (2015).

[7] K. Siemensmeyer, E. Wulf, H.-J. Mikeska, K. Flachbart, S. Gabani, S. Matas, P. Priputen, A. Efdokimova, N. Shitsevalova, Phys. Rev. Lett. 101, 177201 (2008).

[8] F. Iga, A. Shigekawa, Y. Hasegawa, S. Michimura, T. Takabatake, S. Yoshii, T. Yamamoto, M. Hagiwara, K. Kindo, J. Magn. Magn. Mater. 310, e443 (2007).

[9] P. Farkašovský, H. Čenčariková, S. Mat'aš, Phys. Rev. B 82, 054409 (2010). 